\title{
ADSORÇÃO DE HORMÔNIOS EM SOLUÇÃO PRESENTES NOS EFUENTES DA SUINOCULTURA UTILIZANDO CASCA DE ARROZ COMO ADSORVENTE
}

\author{
J. F. HONORIO ${ }^{1 *}$, M. T. VEIT ${ }^{2}$, C. R. G. TAVARES ${ }^{1}$ \\ ${ }^{1}$ Universidade Estadual de Maringá, Departamento de Engenharia Química \\ ${ }^{2}$ Universidade Estadual do Oeste do Paraná, Departamento de Engenharia Química \\ *e-mail: jacqueline-fh@hotmail.com
}

\begin{abstract}
RESUMO
A poluição da água doce no país vem crescendo devido à ineficiência dos tratamentos de efluentes industriais e agropecuários, destinados aos corpos hídricos. Alguns componentes presentes nestes efluentes são desreguladores endócrinos, destacando-se os hormônios naturais. Estas substâncias causam modificações no sistema reprodutivo, como feminização, alterações na transcrição genética, câncer, entre outros. O objetivo do presente trabalho foi o estudo da remoção de hormônios naturais, normalmente presentes nos resíduos gerados nas atividades de suinocultura. $O$ tratamento foi desenvolvido por meio da adsorção de solução de hormônio $17 \beta$-estradiol e estriol, em batelada, utilizando a casca de arroz como adsorvente, sendo este material de origem agroindustrial, abundante no país. A cromatografia líquida de alta eficiência foi empregada para quantificação dos hormônios. Os ensaios de adsorção demonstraram que a remoção dos hormônios não foi influenciada pela a lavagem do adsorvente. A cinética de adsorção apresentou em 2 horas uma quantidade adsorvida em equilíbrio de $0,675 \mathrm{mg} \mathrm{g}^{-1}$ e $0,210 \mathrm{mg} \mathrm{g}^{-1}$ para os hormônios $17 \beta$-estradiol e estriol, respectivamente. A cinética do $17 \beta$-estradiol foi bem descrita pelo modelo pseudo-segunda ordem, e o estriol, por ambos os modelos de pseudo-primeira e pseudo-segunda ordem. O adsorvente demonstrou ser atrativo, pois possui baixo custo, remove os micropoluentes sem necessitar de preparo, com tempo de contato viável para aplicação do tratamento.
\end{abstract}

\section{INTRODUÇÃO}

O Brasil é detentor de uma das maiores reservas mundial de recursos hídricos. A preocupação com o uso, destino e principalmente estado de degradação tem mobilizado pessoas de todas as áreas quanto a sua preservação. Os mananciais são os principais receptores de esgotos, tratados ou não (VERBINNEN et al., 2010).
Contaminantes como produtos farmacêuticos, hormônios naturais e sintéticos e os pesticidas, estão presentes em efluentes municipais e industriais, aos quais se atribui a capacidade de alterar o funcionamento do sistema endócrino (DAMSTRA et al., 2002). Estes contaminantes são capazes de causar efeitos adversos à saúde, afetando o crescimento e reprodução dos seres humanos e animais (BILA e DEZOTTI, 2007). 
O IBGE (2005) destaca o sul do Brasil, como a região em que mais se polui água pelo uso de agrotóxicos, fertilizantes e criação de animais. Isto ocorre porque os estados da região sul do país apresentam sua economia centrada na agricultura e pecuária, como exemplo a suinocultura, que apresenta uma importância econômica muito grande.

De acordo com o Ministério da Agricultura (2012), estudos e investimentos na suinocultura posicionaram o Brasil em quarto lugar no ranking de produção e exportação mundial de carne suína (3,48 mil toneladas de carne suína) destacando a produção de suínos no sul do país, sendo responsável por $61,4 \%$ (ABIPECS, 2012).

Wise et al. (2011) mostraram em seus estudos que as porcas eliminam pela urina cerca de 0,7 a $17 \mathrm{mg}$ por dia de estrogênio quando prenhas. Os estrogênios e progestogênios, naturais ou sintéticos, são excretados pela urina de mamíferos, e uma pequena porção nas fezes, e via efluentes de estações de tratamento, entram em vias aquáticas, podendo causar alterações em organismos aquáticos, tais como feminização ou hermafroditismo (MATTHIESSEN et al., 2002).

Segundo Ghiselli e Jardim (2007) dentre os efeitos adversos causados à saúde humana, são destacados os distúrbios reprodutivos, diferenciação sexual, disfunções de fertilidade, formação de cânceres de testículos, próstata e mama, diminuição na contagem de espermatozoides, endometriose, alterações nos níveis hormonais da tireoide, entre outros.

Os hormônios naturais, como o $17 \beta$ estradiol e $\mathrm{o}$ estriol são os principais causadores de atividades estrogênicas encontradas em corpos hídricos (AERNI et al., 2004), e considerados como responsáveis pela maioria dos efeitos de desregulação endócrina nos organismos (VERBINNEN et al., 2010).

A quantidade gerada de dejetos suínos contendo hormônios naturais como micropoluentes é elevada, uma vez que em média estes animais geram 8,6 litros por dia de dejeto por animal, sendo este valor muito mais expressivo em porcas em gestação e lactação, 16 litros e 27 litros, respectivamente (OLIVEIRA et al., 1993).

Portanto, estes valores de hormônio excretado são bastante expressivos, juntamente com a quantidade de suínos no país, demonstrando a necessidade de estudos e medidas urgentes para o controle e remoção destes contaminantes das águas residuais.

Pesquisas buscam formas de tratamento para remoção dos poluentes na fonte, e tecnologias de aperfeiçoamento para estações de tratamento, tanto de cidades como de indústrias. Segundo Fernandes et al. (2011) os métodos mais utilizados para tratamento de microcontaminantes são aqueles que empregam processos de adsorção e processos oxidativos avançados. Quinlivan et al. (2005) destacam a adsorção dentre as técnicas, pois não resulta em subprodutos, mas tem como agravante o custo dependendo do adsorvente utilizado.

Tentativas têm sido feitas para desenvolver adsorventes baratos utilizando numerosos resíduos agroindustriais (BHATNAGAR e SILLANPÄÄ, 2010). Há estudos em que a atenção tem sido focada na utilização de casca de arroz (MIMURA et al., 2010; HAN et al., 2008) como material adsorvente, com o intuito de agregar valor a este subproduto na remoção de íons metálicos, e corantes têxteis. Este subproduto é gerado em grande volume na região sul do país, onde a produção de arroz é forte economicamente.

Desta forma, o objetivo do presente trabalho é o estudo da remoção $17 \beta$ - estradiol e do estriol em solução, os quais estão presentes nos resíduos gerados nas atividades de suinocultura, por meio da adsorção em batelada utilizando a casca de arroz como material adsorvente alternativo. 


\section{MATERIAIS E MÉTODOS}

\subsection{Reagentes}

Os hormônios utilizados foram estriol (Teor: 97\%) e 17ß-estradiol (Teor: 98\%), da Sigma Aldrich. As soluções padrão de hormônios foram preparadas na concentração de $1000 \mathrm{mg} \mathrm{L}^{-1}$ separadamente, pesando $0,01 \mathrm{~g}$ de analito e dissolvendo em $10 \mathrm{ml}$ de álcool metílico (Teor: 99,8\%) grau HPLC, sendo armazenadas no freezer em frascos âmbar, por no máximo 10 dias. As demais concentrações foram diluídas em água purificada Milli-Q. A acetonitrila (Teor: 99,9\%), grau HPLC, foi utilizada juntamente com água purificada Milli-Q, como fase móvel na quantificação dos hormônios.

\subsection{Metodologia para Quantificação}

A cromatografia liquida de alta eficiência (HPLC) foi empregada na quantificação dos hormônios, sendo a metodologia utilizada validada de acordo com o estabelecido pela Resolução - RE 899, da Agência Nacional de Vigilância Sanitária (ANVISA, 2003) e o DOQ-CGCRE-008, do Instituto Nacional de Metrologia (INMETRO, 2003).

Um equipamento HPLC com detecção UV (Varian 920- LC), equipado com um amostrador automático, uma bomba de gradiente quaternário e com o software de cromatografia Galaxie $^{\mathrm{TM}}$, foi utilizado para leitura de hormônios, sendo acoplado uma coluna HPLC HyperClone ${ }^{\mathrm{TM}}$ ODS (C18) 5 $\mu \mathrm{m}, 250 \mathrm{~mm}$ de comprimento e 4,6 $\mathrm{mm}$ de espessura, e pressão máxima de 3500 psi (Phenomenex). Juntamente a coluna, foi acoplado um Holder Security Guard Universal utilizando cartucho pré-coluna C18 de $4 \mathrm{~mm}$ por $3 \mathrm{~mm}$.

As análises foram feitas com injeção de $20 \mu \mathrm{L}$, sob vazão de $0,7 \quad \mathrm{~mL} \min ^{-1}$ (FERNANDES et al., 2011), no comprimento de onda $225 \mathrm{~nm}$ (CLARA et al., 2010). A eluição foi isocrática com fase móvel acetonitrila:água purificada Milli-Q (45:55 v/v) em leituras com duração de 12 min.

\subsection{Caracterização do Adsorvente}

$\mathrm{O}$ adsorvente casca de arroz in natura, moída, foi gentilmente fornecido por uma agroindústria da região oeste do Paraná. Foi determinado o diâmetro médio de partícula $\left(\overline{D_{s t}}\right)$ e o ponto de carga zero $\left(\mathrm{pH}_{\mathrm{PCZ}}\right)$ para a casca de arroz in natura e após prévia lavagem.

Para a obtenção da biomassa previamente lavada, uma quantidade de casca de arroz foi submetida a um processo lavagem com três enxágues em água purificada Milli-Q e secagem em estufa com circulação de ar na temperatura de $50{ }^{\circ} \mathrm{C}$ por 24 horas.

\subsubsection{Distribuição Granulométrica}

Uma quantidade de $200 \mathrm{~g}$ de biomassa, foi colocada no topo de um conjunto de peneiras $(12,14,16,20,28,32,35,42,48,80$, 100, 200, 270 e 325 mesh, marca Bertel) sobre o equipamento vibrador, sobrepondo-as em ordem crescente de abertura das malhas e tampadas, durante $15 \mathrm{~min}$ de peneiramento (GOMIDE, 1983).

Para cada peneira, a massa foi pesada e a porcentagem do adsorvente retido em cada peneira foi calculada conforme Equação 1.

$$
\% \mathrm{R}=(\mathrm{PRi} \times 100) / \mathrm{P}_{\mathrm{t}}
$$

Em que $\% \mathrm{R}$ é a porcentagem retida em cada peneira, $\mathrm{P}_{\mathrm{t}}$ é o peso da amostra em gramas e PRi é o peso retido na peneira $\mathrm{i}$.

Determinadas as frações, a biomassa foi caracterizada quanto ao tipo do sólido particulado (GOMIDE, 1983) e o diâmetro de Sauter (MASSARANI, 1984).

Segundo Gomide (1983) os sólidos particulados podem ser classificados em cinco tipos, sendo pós as partículas de $1 \mu$ até 0,5 $\mathrm{mm}$, sólidos granulares $(0,5 \mathrm{a} 10 \mathrm{~mm})$ e blocos acima de $1 \mathrm{~cm}$. 
De acordo com Massarani (1984) o diâmetro médio de Sauter $\left(\overline{D_{s t}}\right)$ pode ser determinado conforme a Equação 2, 3 e 4.

$$
\begin{gathered}
\overline{D_{s t}}=\frac{1}{\sum\left(\frac{\Delta X_{i}}{\overline{D_{i .}}}\right)_{i}} \\
\overline{D_{i}}=\frac{D_{i}-D_{i-1}}{2} \\
\Delta X_{i}=\frac{m_{i}}{M}
\end{gathered}
$$

Em que $\left(\overline{D_{s t}}\right)$ é o diâmetro médio de Sauter, $\left(\Delta \mathrm{X}_{\mathrm{i}}\right)$ è a fração retida na peneira $\mathrm{i}$, $\left(\bar{D}_{i}\right)$ é o diâmetro médio entre as peneiras i e i1, $\left(D_{\mathrm{i}}\right)$ é o diâmetro da peneira $\mathrm{i},\left(\mathrm{D}_{\mathrm{i}-1}\right)$ é o diâmetro da peneira de abertura maior superior a peneira $\mathrm{i},\left(\mathrm{m}_{\mathrm{i}}\right)$ é o peso da amostra retida sobre a peneira $\mathrm{i}(\mathrm{g})$ e (M) é o peso total da amostra $(\mathrm{g})$.

\subsubsection{Ponto de carga zero (PCZ)}

$\mathrm{O}$ procedimento utilizado para determinação do ponto de carga zero foi descrito por Regalbuto e Robles (2004), denominada "experimento dos 11 pontos". Para tanto, utilizou-se $50 \mathrm{mg}$ do adsorvente, granulometria mista, com $50 \mathrm{~mL}$ de solução aquosa, sob diferentes condições de $\mathrm{pH}$ inicial $(2,3,4,5,6,7,8,9,10,11,12)$, ajustados com soluções de $\mathrm{HCl}$ ou $\mathrm{NaOH} 1 \mathrm{~mol} \mathrm{~L}^{-1}$, à temperatura ambiente $\left(25-30^{\circ} \mathrm{C}\right)$, com agitação de $180 \mathrm{rpm}$ em incubadora com agitação mecânica orbital, por $24 \mathrm{~h}$ (GUILARDUCI et al., 2006; MIMURA et al., 2010). O pH final foi determinado para cada ponto, sendo todos em duplicata. $\mathrm{O} \mathrm{pH}_{\mathrm{PCZ}}$ corresponde a média dos pontos na qual o $\mathrm{pH}$ final se mantém constante, independentemente do $\mathrm{pH}$ inicial, ou seja, a superfície se comporta como um tampão.

\subsection{Ensaios de Adsorção}

Foram realizados ensaios de adsorção com 17ß-estradiol e estriol em biomassa casca de arroz para a avaliação da influência da lavagem da biomassa, bem como ensaios de cinética de adsorção para a determinação do tempo de contato, sendo as cinéticas também representadas por modelos da literatura.

\subsubsection{Influência da lavagem da biomassa}

A avaliação da influência da lavagem da biomassa na adsorção, foi realizada com $0,2 \mathrm{~g}$ de casca de arroz in natura e a mesma massa de casca de arroz previamente lavada, colocadas em contato com $25 \mathrm{~mL}$ de solução de $17 \beta$-estradiol $\left(7 \mathrm{mg} \mathrm{L}^{-1}\right)$, e analogamente para o estriol $\left(7 \mathrm{mg} \mathrm{L}^{-1}\right)$. Os frascos foram mantidos sob agitação orbital de $100 \mathrm{rpm}$ (garantindo mistura da biomassa), temperatura ambiente $\left(25-35^{\circ} \mathrm{C}\right)$ por $20 \mathrm{~h}$. Posteriormente, as amostras foram filtradas em membranas $0,45 \mu \mathrm{m}, \quad$ e quantificadas conforme metodologia definida em HPLC-UV. A quantidade de cada hormônio removido pelo adsorvente foi calculada empregando a Equação 5.

$$
q_{e q}=\frac{\left(C_{o}-C\right) * V}{m}
$$

Em que, $\mathrm{C}_{0}$ é a concentração inicial de analito (mg $\left.\mathrm{L}^{-1}\right) ; \quad \mathrm{C}$ é a concentração remanescente do analito $\left(\mathrm{mg} \mathrm{L}^{-1}\right)$; q $\mathrm{q}_{\mathrm{eq}}$ é a quantidade adsorvida de analito no equilíbrio $\left(\mathrm{mg} \mathrm{g}^{-1}\right)$; $\mathrm{m}$ é a massa de adsorvente $(\mathrm{g})$ em base seca, e V é o volume da solução de analito em contato com o adsorvente (L).

\subsubsection{Cinética de Adsorção}

O tempo de contato foi obtido pela cinética de adsorção, em que $0,3 \mathrm{~g}$ da biomassa in natura foram postas em contato com $25 \mathrm{~mL}$ de solução sob agitação de 100 rpm e temperatura de $25^{\circ} \mathrm{C}$ na concentração 10 $\mathrm{mg} \mathrm{L}^{-1}$ para o $17 \beta$-estradiol e $5 \mathrm{mg} \mathrm{L}^{-1}$ para o estriol. Em intervalos regulares de tempo até 4 
horas, foram retiradas amostras em duplicata, filtradas em membranas $0,45 \mu \mathrm{m}$, quantificadas conforme metodologia definida em HPLC-UV e determinadas às quantidades adsorvidas conforme Equação 6.

$$
q(t)=\frac{\left(C_{o}-C(t)\right) * V}{m}
$$

Em que, $(\mathrm{C}(\mathrm{t}))$ é a concentração remanescente de analito no tempo $(\mathrm{t})\left(\mathrm{mg} \mathrm{L}^{-1}\right)$ e (q (t)) é a quantidade adsorvida de analito no tempo $(\mathrm{t})\left(\mathrm{mg} \mathrm{g}^{-1}\right)$.

Aos dados da cinética de adsorção foram aplicados os modelos de pseudo-primeira ordem (Equação 7) e pseudo-segunda ordem (Equação 8), descritos por Lagergren (1898) e Ho e Mckay (1998), respectivamente.

$$
\begin{aligned}
& q(t)=q_{e q}\left(1-e^{-k_{1} t}\right) \\
& q(t)=q_{e q} \frac{q_{e q} k_{2} t}{q_{e q} k_{2} t+1}
\end{aligned}
$$

Em que, $\mathrm{k}_{1}$ é a constante da taxa de adsorção do modelo pseudo-primeira ordem $\left(\min ^{-1}\right)$ e $k_{2}$ é a constante da taxa de adsorção de pseudo-segunda ordem $\left(\mathrm{g} \mathrm{mg}^{-1} \mathrm{~min}^{-1}\right)$.

Os parâmetros dos modelos foram obtidos usando o software Statistica para Windows ${ }^{\circledR}$, versão 7.0, o método não-linear simplex com função objetivo determinada pela soma dos erros (observada-previsto) ${ }^{2}$.

\section{RESULTADOS E DISCUSSÕES}

\subsection{Caracterização do adsorvente}

A determinação do diâmetro médio de partícula e do ponto de carga zero, foram realizadas para caracterizar a granulometria utilizada do adsorvente, bem como determinar o pH dos ensaios de adsorção.

\subsubsection{Distribuição Granulométrica}

A Tabela 1 apresenta as porcentagens da casca arroz, in natura e lavada distribuídas nas peneiras para determinação do tamanho de partícula.
Tabela 1 - Porcentagem de biomassa retida nas peneiras.

\begin{tabular}{cccc}
\hline Mesh & Diâmetro & \multicolumn{2}{c}{ \% retida } \\
\cline { 3 - 4 } médio $(\mathrm{mm})$ & in natura & Lavada \\
\hline$>12$ & 1,4 & 0 & 0,49 \\
$12-14$ & 1,29 & 0 & 0,18 \\
$14-16$ & 1,09 & 0,33 & 0,81 \\
$16-20$ & 0,925 & 8,05 & 13,39 \\
$20-28$ & 0,725 & 33,70 & 37,41 \\
$28-32$ & 0,55 & 26,50 & 25,21 \\
$32-35$ & 0,4625 & 0,24 & 0,56 \\
$35-42$ & 0,39 & 2,88 & 2,081 \\
$42-48$ & 0,3275 & 7,03 & 5,54 \\
$48-80$ & 0,24 & 12,15 & 10,47 \\
$80-100$ & 0,165 & 0,93 & 0,89 \\
$100-200$ & 0,1125 & 3,31 & 1,86 \\
$200-270$ & 0,064 & 1,12 & 0,44 \\
$270-325$ & 0,049 & 0,85 & 0,25 \\
$>325$ & 0,045 & 2,91 & 0,43 \\
\hline
\end{tabular}

Fonte: Própria.

$\mathrm{Na}$ Tabela 1 se pode verificar que a casca de arroz in natura tem uma grande quantidade de partículas mais finas, sendo classificado 30,4\% como pó (GOMIDE,1983). Esta porcentagem de pó é reduzida para $22,5 \%$ quando realizada uma prévia lavagem e secagem da biomassa.

Em torno de $60 \%$ da biomassa, tanto na forma in natura como lavada, estão retidas nas peneiras com diâmetro entre 0,5 e 0,85 mm. Segundo Massarani (1984), o Diâmetro de Sauter, que caracteriza o diâmetro médio da partícula, apresentou para a casca de arroz in natura diâmetro médio de $0,39 \mathrm{~mm}$, e para a casca de arroz lavada um diâmetro médio de $0,47 \mathrm{~mm}$. Os ensaios de adsorção foram realizados com granulometria mista.

\subsubsection{Ponto de Carga Zero (PCZ)}

O resultado obtido para o PCZ da casca de arroz é apresentado na Figura 1 . O valor do $\mathrm{pH}_{\mathrm{PCZ}}$ foi de 5 a 10 , onde os $\mathrm{pH}$ finais se 
mantiveram constantes independente do $\mathrm{pH}$ inicial.

Figura 1 - Ponto de carga zero do adsorvente casca de arroz in natura e lavado.

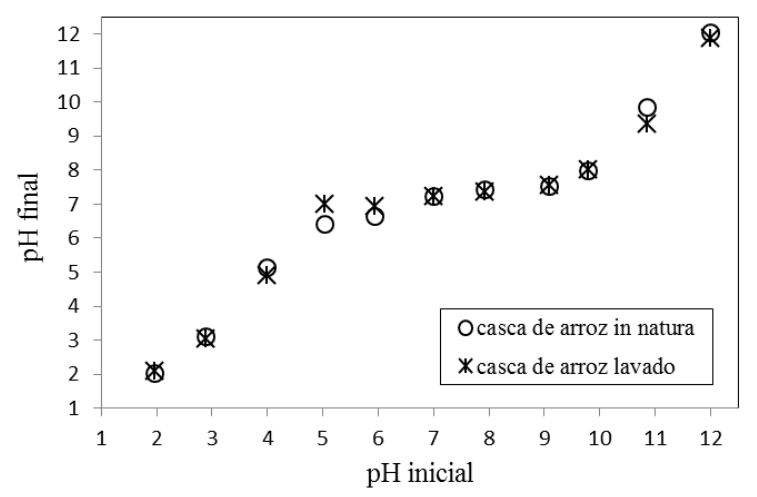

Fonte: Própria.

A Figura 1 demonstrou pequenas diferenças na faixa de $\mathrm{pH}$ constante para a biomassa in natura ou submetida à lavagem, sendo verificada a sobreposição de grande parte dos pontos. $\mathrm{O}$ cálculo do $\mathrm{pH}_{\mathrm{PCZ}}$ foi realizado pela média aritmética dos pontos que se apresentaram constantes para o $\mathrm{pH}$ final, pois esta faixa apresenta comportamento tampão em solução aquosa (REGALBUTO e ROBLES, 2004). A casca de arroz apresentou $\mathrm{pH}_{\mathrm{PCZ}}$ próximos, sendo 7,20 para a casca de arroz in natura e 7,34 para a casca de arroz lavada. Estes resultados aproximam-se do obtido por Mimura et al. (2010), que obteve em seus estudos de adsorção de metais em casca de arroz $\mathrm{pH}_{\mathrm{PCZ}}$ de 7. Os ensaios de adsorção foram realizados em $\mathrm{pH}$ neutro próximo ao $\mathrm{pH}_{\mathrm{PCZ}}$ da casca de arroz para verificar o comportamento do adsorvente na remoção dos poluentes sem alteração de $\mathrm{pH}$.

\subsection{Ensaios de Adsorção}

Nos ensaios de adsorção foi avaliada a necessidade da lavagem do adsorvente para verificar a melhora na remoção dos hormônios $17 \beta$-estradiol e estriol. A cinética de adsorção determinou o tempo de contato para que o sistema atingisse equilíbrio.

\subsubsection{Influência da Lavagem da Biomassa}

A influência da biomassa submetida a uma lavagem em relação à biomassa in natura foi avaliada por um processo de adsorção na remoção dos hormônios 17ß-estradiol e estriol, conforme apresentado na Figura 2. Esses ensaios permitiram verificar a necessidade da lavagem da biomassa (gastos adicionais) para aumentar a quantidade adsorvida dos hormônios no processo.

Figura 2 - Adsorção com biomassa in natura e lavada. (dosagem biomassa $=8 \mathrm{~g} \mathrm{~L}^{-1}, \mathrm{C}_{0}=7 \mathrm{mg} \mathrm{L}^{-1}$, $100 \mathrm{rpm}, 25-35^{\circ} \mathrm{C}, 20$ horas)

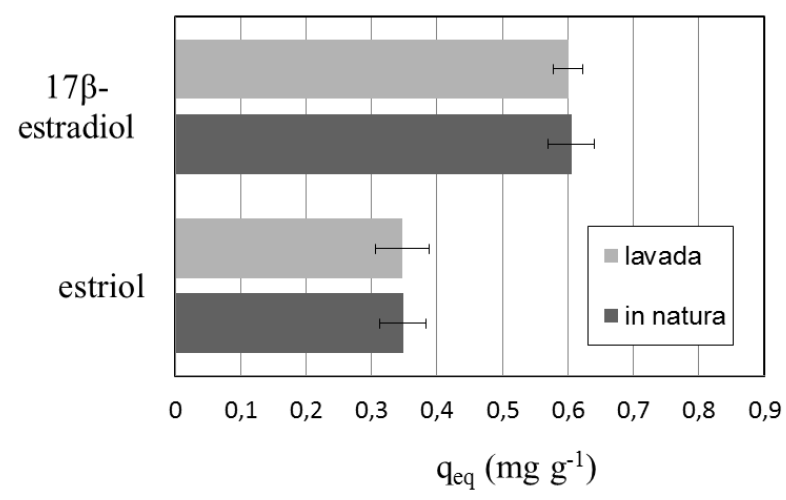

Fonte: Própria.

Observa-se na Figura 2 que não houve mudanças na quantidade adsorvida em equilíbrio para as biomassas in natura $\mathrm{e}$ lavada, com ambos os hormônios. Para o 17ßestradiol, os qeq foram de $0,605 \mathrm{mg} \mathrm{g}^{-1}$ e 0,600 $\mathrm{mg} \mathrm{g}^{-1}$, referente a casca de arroz in natura $\mathrm{e}$ lavada, respectivamente. $\mathrm{O}$ estriol apresentou $\mathrm{q}_{\mathrm{eq}}$ de $0,348 \mathrm{mg} \mathrm{g}^{-1}$ para a biomassa in natura e $0,347 \mathrm{mg} \mathrm{g}^{-1}$ para a biomassa lavada.

Este estudo para a remoção dos dois hormônios comprovou que a casca de arroz não apresenta sujeiras passiveis de remoção em água interferentes no processo de adsorção, não justificando gastos com lavagem da biomassa. Assim, as cinéticas de adsorção foram realizadas com biomassa in natura. 


\subsection{Cinética de Adsorção}

O tempo de equilibrio da adsorção dos hormônios em casca de arroz in natura bem como os modelos cinéticos são apresentados nas Figuras 3-a,b.

Figura 3 - Cinética de adsorção (dosagem biomassa $=12 \mathrm{~g} \mathrm{~L}^{-1}, 100 \mathrm{rpm}, 25^{\circ} \mathrm{C}, 4$ horas)

(a) 17 $\beta$-estradiol $\left(\mathrm{C}_{0}=10 \mathrm{mg} \mathrm{L}^{-1}\right)$

(b) estriol $\left(\mathrm{C}_{0}=5 \mathrm{mg} \mathrm{L}^{-1}\right)$

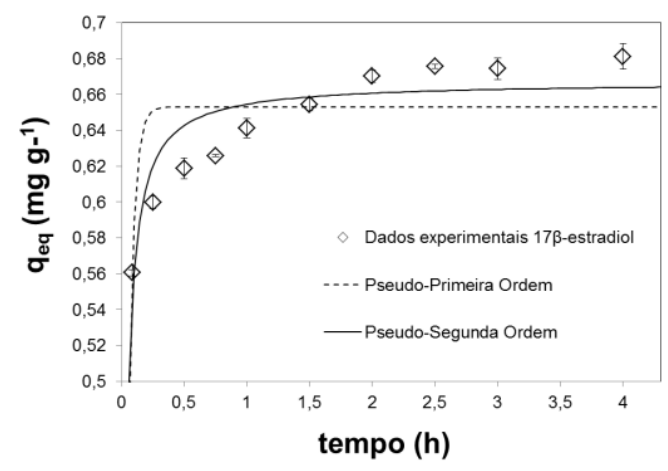

(a)

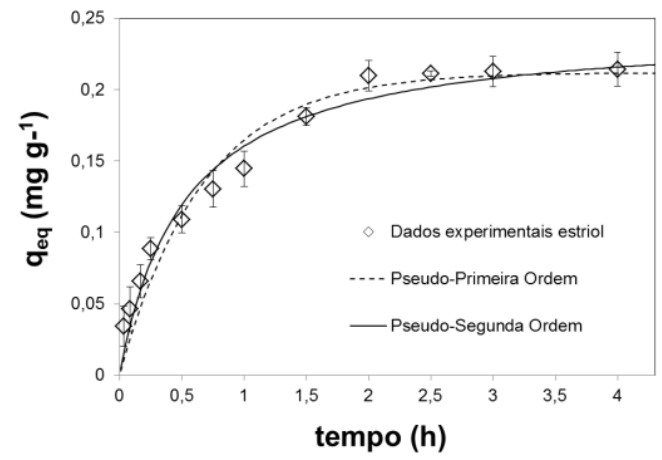

(b)

Fonte: Própria.

As cinéticas de remoção dos hormônios $17 \beta$-estradiol e estriol apresentaram tempo de equilíbrio de 2 horas, conforme se pode observar nas Figuras 3-a,b. O 17ß-estradiol atingiu $\mathrm{q}_{\mathrm{eq}}$ de $0,675 \mathrm{mg} \mathrm{g}^{-1}$ e o estriol 0,210 $\mathrm{mg} \mathrm{g}^{-1}$.

Em relação ao tempo de contato, a casca de arroz demonstrou ser rápida na remoção do 17ß-estradiol comparada com a obtida por Fernandes et al. (2011) que em seus estudos obtiveram tempo de contato superiores a 7 horas na remoção do hormônio estradiol. Os autores atingiram $\mathrm{q}_{\mathrm{eq}}$ 0,086-0,200 $\mathrm{mg} \mathrm{g}^{-1}$ empregando como material adsorvente a turfa decomposta na concentração inicial de 0,10 $\mathrm{mg} \mathrm{L}^{-1}$, em contato com 50, 75, 100, 150 e 200 mg de turfa.

Zhang e Zhou (2005) estudaram vários materiais adsorventes para a remoção do $17 \beta$ estradiol e obtiveram maior remoção com o adsorvente carbonáceo $\left(7,0 \mu \mathrm{g} \mathrm{g}^{-1}\right)$ em 7 horas e seguida do carvão ativado $\left(0,5 \mu \mathrm{g} \mathrm{g}^{-1}\right) \mathrm{em}$ $25 \mathrm{~h}$, apresentando também um tempo de contato bastante superior ao da casca de arroz.

Os resultados obtidos no presente trabalho demonstraram a grande capacidade de remoção do 17-estradiol em casca de arroz quando comparado os valores de $\mathrm{q}_{\mathrm{eq}}$ com os obtidos em outros estudos apresentados. Os resultados obtidos para os parâmetros dos modelos pseudo-primeira e pseudo-segunda ordem são apresentados na Tabela 2.

Tabela 2 - Modelos cinéticos de pseudo-primeira e pseudo-segunda ordem, para ajustes dos dados experimentais de adsorção dos hormônios $17 \beta$ estradiol e estriol, com adsorvente casca de soja.

\begin{tabular}{|c|c|c|}
\hline & $17 \beta$-estradiol & Estriol \\
\hline qeq experimenta & $0,675 \pm 0,004$ & $0,210 \pm 0,010$ \\
\hline \multicolumn{3}{|c|}{ Pseudo-Primeira Ordem } \\
\hline $\mathrm{q}_{\text {eq calculado }}$ & 0,653 & 0,212 \\
\hline $\mathrm{k}_{1}$ & 23,081 & 1,501 \\
\hline $\mathrm{R}^{2}$ & 0,991 & 0,983 \\
\hline \multicolumn{3}{|c|}{ Pseudo-Segunda Ordem } \\
\hline $\mathrm{q}_{\text {eq calculado }}$ & 0,667 & 0,244 \\
\hline $\mathrm{k}_{2}$ & 79,046 & 7,866 \\
\hline $\mathrm{R}^{2}$ & 0,996 & 0,989 \\
\hline
\end{tabular}

Fonte: Própria.

De acordo com a Tabela 2, juntamente com a Figura 3-a, o modelo de pseudosegunda ordem representou melhor os dados da cinética de adsorção do hormônio $17 \beta$ estradiol apresentando $\mathrm{q}_{\mathrm{eq}}$ calculado $(0,667 \mathrm{mg}$ $\left.\mathrm{g}^{-1}\right)$ próximo ao obtido experimentalmente $\left(0,675 \mathrm{mg} \mathrm{g}^{-1}\right)$, e apresentando o melhor coeficiente de correlação $(0,996)$. Este modelo pressupõe que as interações físico-químicas entre o adsorvato e o grupo da superfície do 
adsorvente são a etapa determinante da velocidade de reação (MIMURA et al., 2010).

Observando a cinética de adsorção do estriol (Figura 3-b), ambos os modelos representam bem os dados. $\mathrm{O}$ modelo de pseudo-primeira ordem foi o que obteve o q calculado $\left(0,212 \mathrm{mg} \mathrm{g}^{-1}\right)$ aproximadamente igual ao obtido experimentalmente $(0,210 \mathrm{mg}$ $\mathrm{g}^{-1}$ ), mas o modelo de pseudo-segunda ordem apresentou melhor coeficiente de correlação.

Sendo assim, a adsorção do estriol envolve o proposto pelos dois modelos, sendo tanto as interações físico-químicas entre o adsorvato e o adsorvente proposto pelo modelo pseudo-segunda ordem, bem como o número de sítios disponíveis para a adsorção (MIMURA et al., 2010), proposto pelo modelo pseudo-primeira ordem limitantes no processo de adsorção do estriol, diferente do ocorrido com o hormônio $17 \beta$-estradiol, descrito apenas pelo modelo de pseudo- segunda ordem. Estes modelos apesar de serem comumente utilizados, são satisfatórios no esclarecimento dos processos envolvidos na adsorção.

\section{CONCLUSÃO}

Estudos propondo tratamentos de microcontaminantes como o $17 \beta$-estradiol e estriol, hormônios resultantes de atividades pecuárias, apresentam grande relevância. Estas pesquisas proporcionam aos órgãos regulamentadores, a visão de novos poluentes de grande impacto, tanto na saúde como ao ambiente. Além disso, estes estudos propõem também, tratamentos de baixo custo para estes contaminantes ainda negligenciados.

$\mathrm{O}$ presente trabalho demonstrou que a casca de arroz é um adsorvente promissor na remoção dos hormônios $17 \beta$-estradiol e estriol. A adsorção não necessita de gastos com lavagem para melhorar o processo, e apresentou um tempo de contato ( 2 horas) viável para aplicação em tratamentos, atingindo uma boa quantidade adsorvida em equilíbrio dos hormônios.

$\mathrm{O}$ resíduo líquido da suinocultura, em sua grande parte é destinado à aplicação em solo como fertilizante. A adsorção dos hormônios $17 \beta$-estradiol e estriol em casca de arroz permite $\mathrm{o}$ tratamento destes contaminantes sem alterar o fato da aplicação direta em solo, pois este adsorvente é composto em sua grande parte por matéria orgânica. $\mathrm{O}$ hormônio adsorvido na superfície do adsorvente, sob ação do sol nas lavouras, sofre degradação restando apenas a casca de arroz que se torna também biofertilizante.

\section{NOMENCLATURA}

C Concentração remanescente do analito $\left(\mathrm{mg} \mathrm{L}^{-1}\right)$

$\mathrm{C}_{0} \quad$ Concentração inicial de analito $\left(\mathrm{mg} \mathrm{L}^{-1}\right)$

$\mathrm{C}(\mathrm{t}) \quad$ Concentração remanescente de analito no tempo $(\mathrm{t})\left(\mathrm{mg} \mathrm{L}^{-1}\right)$

$\overline{D_{i}} \quad$ Diâmetro médio entre as peneiras i (mm)

$\mathrm{D}_{\mathrm{i}} \quad$ Diâmetro da peneira i $(\mathrm{mm})$

$\mathrm{D}_{\mathrm{i}-1} \quad$ Diâmetro da peneira de abertura maior superior a peneira i $(\mathrm{mm})$

$\overline{D_{s t}} \quad$ Diâmetro médio de Sauter (mm)

$\mathrm{k}_{1} \quad$ Constante da taxa de adsorção do modelo pseudo-primeira ordem $\left(\mathrm{min}^{-1}\right)$

$\mathrm{k}_{2}$ Constante da taxa de adsorção de pseudo segunda ordem $\left(\mathrm{g} \mathrm{mg}^{-1} \mathrm{~min}^{-1}\right)$

$\mathrm{m} \quad$ Massa de adsorvente ( $\mathrm{g}$ ) em base seca

$m_{i} \quad$ Peso da amostra retida sobre a peneira i (g)

M Peso total da amostra (g)

$\mathrm{pH}_{\mathrm{PCZ}} \quad \mathrm{pH}$ no ponto de carga zero

PRi Peso retido na peneira i $(\mathrm{g})$

$\mathrm{P}_{\mathrm{t}} \quad$ Peso da amostra (g)

$\mathrm{q}_{\mathrm{eq}} \quad$ Quantidade adsorvida de analito no equilíbrio $\left(\mathrm{mg} \mathrm{g}^{-1}\right)$

$\mathrm{q}(\mathrm{t})$ Quantidade adsorvida de analito no tempo (t) $\left(\mathrm{mg} \mathrm{g}^{-1}\right)$

$\mathrm{R}^{2} \quad$ Coeficiente de determinação 
V Volume da solução de analito em contato com o adsorvente (L)

$\Delta \mathrm{X}_{\mathrm{i}} \quad$ Fração retida na peneira $\mathrm{i}$

$\% \mathrm{R}$ Porcentagem retida em cada peneira

\section{REFERÊNCIAS}

ABIPECS: Disponível em: $<$ http://www.abipecs.org.br/uploads/relatori os/relatorios-

associados/ABIPECS_relatorio_2012_pt.pdf >. Acesso em: julho 2014.

AERNI, H.R.; KOBLER, B.; RUTISHAUSER, B.V.; WETTSTEIN, F.E.; FISCHER, R.; GIGER, W.; HUNGERBÜHLER, A.; MARAZUELA, M.D.; PETER, A.; SCHÖNEN-BERGER, R.; VÖGELI, A.C.; SUTER, M.J.F.; EGGEN, R.I.L. Combined biological and chemical assessment of estrogenic activities in wastewater treatment plant effluents. Analytical Bioanalytical Chemistry. v. 78, p.688-696, 2004.

ANVISA. Agencia Nacional de Vigilância Sanitária Resolução - RE n 899, de29 de maio de 2003. Guia para validação de métodos analíticos e bioanalíticos. Brasilia, 2003.

BHATNAGAR, A; SILLANPÄÄ, M. Utilization of agro-industrial and municipal waste materials as potential adsorbents for water treatment-A review. Chemical Engineering Journal. v. 157, p.277-296, 2010.

BILA, D.M.; DEZOTTI, M. Desreguladores Endócrinos no Meio Ambiente: Efeitos e Consequências. Química Nova. v. 30, n. 3, p. 651-666, 2007.

CLARA, M.; STRENN, B.; SARACEVIC, E.; KREUZINGER, N. Adsorption of bisphenol-
A, $17 \beta$-estradiole and $17 \alpha$-ethinylstradiole to sewage sludge. Chemosphere. v.56, p. 843851, 2004.

DAMSTRA T.; BARLOW S.; BERGMAN A.; KAVLOCK R.; VAN DER KRAAK G. Global Assessment of The State-Of-TheScience of Endocrine Disruptors; International Programme On Chemical Safety; WHO; Geneva; 515p, 2002.

FERNANDES, A.N.; GIOVANELA, M; CARLOS A.P.; ESTEVES, V.I.; SIERRA, M.M.D.; GRASSI, M.T. Remoção dos Hormônios $17 \beta$-estradiol e $17 \alpha$-etinilestradiol de soluções aquosas empregando turfa decomposta como material adsorvente. Química Nova. v. 34, n.9, p.1526-1533, 2011.

GHISELLI, G.; JARDIM, F. W. Interferentes Endócrinos no Ambiente. Química Nova. v. 30, n. 3, p. 695-706, 2007.

GOMIDE, R. Operações Unitárias. Operações com sistemas sólidos Granulares. São Paulo, v. 1, p. 13-43, 1983.

GUILARDUCI, V.V.S.; MESQUITA， J.P.; MARTELLI, P.B.; GORGULHO, H.F. Adsorção de fenol sobre carvão ativado em meio alcalino. Química Nova. v. 29, n. 6, p. 1226-1232, 2006.

HAN, R.P.; DING, D.D.; XU, Y.F.; ZOU, W.H.; WANG, Y.F.; LI, Y.F.; ZOU,L. Use of rice husk for the adsorption of congo red from aqueous solution in column mode. Bioresource Technology. v. 99, p.2938-2946, 2008.

HO, Y.S.; MCKAY, G. Kinetic models for the sorption of dye from aqueous solution by wood. Trans IChemE, v. 76B, p. 183-191, 1998. 
HO, Y.S. Review of second-order models for adsorption systems. Jornal of Hazardous Materials, v. B136, p. 681-689, 2006.

IBGE - Perfil dos municípios brasileiros: meio ambiente. Rio de Janeiro, 2005. Disponível em:

$<$ http://www.ibge.gov.br/home/estatística/ec onomia/perfilmunic/meio_ambiente_2002/m eio_ambiente2002.pdf defoult.shtm>.

Acesso em: 02 maio 2013.

INMETRO, 2003. Disponivel em: $<$ http://www.inmetro.gov.br/Sidoq/Arquivos /CGCRE/DOQ/DOQ-CGCRE-8_02.pdf> .

Acesso em: 15/09/2014.

LAGERGREN, S. Zur theorie der sogenannten adsorption gelösterstoffe. Bihang TillKungligaSvenskaVetenskaps-

akademiens. Handlingar, Band, 24, afd.II, no.4, p. 1-39, 1898.

MASSARANI, G. Problemas em sistemas particulados. São Paulo. Editora Edgard Blucher LTDA. p. 2,3, 1984.

MATTHIESSEN, P.; ALLEN, Y.; BAMBER, S.; CRAFT, J.; HURST, M.; HUTCHINSON, T.; FEIST, S.; KATSIADAKI, I.; KIRBY, M.; ROBINSON, C.; SCOTT, S.; THAIN, J.; THOMAS, K. The impact of oestrogenic and androgenic comtamination on marine organisms in the United Kingdom-summary of the EDMAR programme. Endocrine disruption in the marine environment. Marine Environmental Research, v.54, p.645-649, 2002.

MIMURA, A.M.S.; VIEIRA, T.V.A.; MARTELLI, P.B.; GORGULHO, H.F. Aplicação da casca de arroz na adsorção dos íons $\mathrm{Cu}^{2+}, \mathrm{Al}^{3+}, \mathrm{Ni}^{2+}$ e $\mathrm{Zn}^{2+}$. Química Nova. v. 33, n. 6, p.1279-1284, 2010.
MINISTÉRIO DA AGRICULTURA, 2012.

Disponível em: $<$ http://www.agricultura.gov.br/animal/espe cies/suinos $>$. Acesso em: 12/11/2012.

OLIVEIRA, P.A.V. de. (Coord.) Manual de manejo e utilização dos dejetos de suínos. Concórdia: EMBRAPA-CNPSA, 1993. 188p. (EMBRAPA-CNPSA. Documentos, 27)

QUINLIVAN, P.A.; LI, L.; KNAPPE, D.R.U.; Effects of activated carbon characteristics on the simultaneous adsorption of aqueous organic micropollutants and natural organic matter. Water Research. v. 39, p.1663-1673, 2005.

REGALBUTO, J.R.; ROBLES, J. The engineering of Pt/Carbon Catalyst Preparation - For application on Proton Exchange Fuel Cell Membrane (PEFCM). University of Illinois, Chicago. p.4-7, 2004.

VERBINNEN, R.T., NUNES, G.S., VIEIRA, E.M. Determinação de hormônios estrógenos em agua potável usando CLAE-DAD. Química Nova. v. 33, n. 9, p. 1837-1842, 2010.

WISE, A., O'BRIEN, K., WOODRUFF, T. Are Oral Contraceptives a Significant Contributor to the Estrogenicity of Drinking Water? Environmental Science Technology. v. 45, p.51-60, 2011.

ZHANG, Y., ZHOU, J.L. Removal of estrone and 17b- stradiol from water by adsorption.Water Research, v.39, p.39914003, 2005.

\section{AGRADECIMENTOS}

O presente trabalho foi realizado com o apoio da Coordenação de Aperfeiçoamento de Pessoal de Nível Superior - CAPES - Brasil. 